University of Wollongong

Research Online

Faculty of Engineering and Information

Faculty of Engineering and Information

Sciences - Papers: Part B

Sciences

2020

\title{
Validation of Geant4 for silicon microdosimetry in heavy ion therapy
}

David Bolst

University of Wollongong, dbolst@uow.edu.au

Susanna Guatelli

University of Wollongong, susanna@uow.edu.au

Linh T. Tran

University of Wollongong, tltran@uow.edu.au

Lachlan Chartier

University of Wollongong, Ic752@uowmail.edu.au

Jeremy A. Davis

University of Wollongong, jeremyd@uow.edu.au

See next page for additional authors

Follow this and additional works at: https://ro.uow.edu.au/eispapers1

Part of the Engineering Commons, and the Science and Technology Studies Commons

Research Online is the open access institutional repository for the University of Wollongong. For further information contact the UOW Library: research-pubs@uow.edu.au 


\title{
Validation of Geant4 for silicon microdosimetry in heavy ion therapy
}

\begin{abstract}
Microdosimetry is a particularly powerful method to estimate the relative biological effectiveness (RBE) of any mixed radiation field. This is particularly convenient for therapeutic heavy ion therapy (HIT) beams, referring to ions larger than protons, where the RBE of the beam can vary significantly along the Bragg curve. Additionally, due to the sharp dose gradients at the end of the Bragg peak (BP), or spread out BP, to make accurate measurements and estimations of the biological properties of a beam a high spatial resolution is required, less than a millimetre. This requirement makes silicon microdosimetry particularly attractive due to the thicknesses of the sensitive volumes commonly being $\sim 10$ [Formula: see text]m or less. Monte Carlo (MC) codes are widely used to study the complex mixed HIT radiation field as well as to model the response of novel microdosimeter detectors when irradiated with HIT beams. Therefore it is essential to validate $M C$ codes against experimental measurements. This work compares measurements performed with a silicon microdosimeter in mono-energetic [Formula: see text], [Formula: see text] and [Formula: see text] ion beams of therapeutic energies, against simulation results calculated with the Geant4 toolkit. Experimental and simulation results were compared in terms of microdosimetric spectra (dose lineal energy, [Formula: see text]), the dose mean lineal energy, y D and the RBE10, as estimated by the microdosimetric kinetic model (MKM). Overall Geant4 showed reasonable agreement with experimental measurements. Before the distal edge of the BP, simulation and experiment agreed within $\sim 10 \%$ for $y \mathrm{D}$ and $\sim 2 \%$ for RBE10. Downstream of the BP less agreement was observed between simulation and experiment, particularly for the [Formula: see text] and [Formula: see text] beams. Simulation results downstream of the BP had lower values of $y$ D and RBE10 compared to the experiment due to a higher contribution from lighter fragments compared to heavier fragments.
\end{abstract}

\section{Disciplines}

Engineering | Science and Technology Studies

\section{Publication Details}

Bolst, D., Guatelli, S., Tran, L. T., Chartier, L., Davis, J., Biasi, G., Prokopovich, D. A., Pogossov, A., Reinhard, M. I., Petasecca, M., Lerch, M. L. F., Matsufuji, N., Povoli, M., Summanwar, A., Kok, A., Jackson, M. \& Rosenfeld, A. B. (2020). Validation of Geant4 for silicon microdosimetry in heavy ion therapy. Physics in medicine and biology, 65 (4), 045014.

\section{Authors}

David Bolst, Susanna Guatelli, Linh T. Tran, Lachlan Chartier, Jeremy A. Davis, Giordano Biasi, Dale A. Prokopovich, Alex Pogossov, Mark I. Reinhard, Marco Petasecca, Michael L. F Lerch, N Matsufuji, M Povoli, A Summanwar, Angela Kok, Michael A. Jackson, and Anatoly B. Rosenfeld 


\title{
Validation of Geant4 for silicon microdosimetry in heavy ion therapy
}

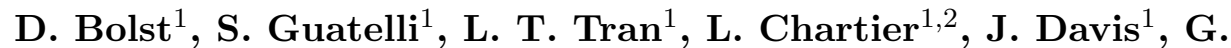

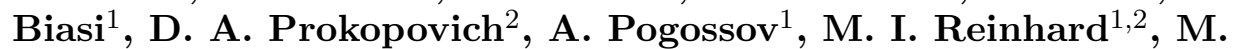 \\ Petasecca $^{1}$, M. L. F. Lerch ${ }^{1}$, N. Matsufuji ${ }^{3}$, M. Povoli ${ }^{4}$, A. \\ Summanwar $^{4}$, A. Kok ${ }^{4}$, M. Jackson ${ }^{5}$ and A. B. Rosenfeld ${ }^{1}$ \\ ${ }^{1}$ Centre for Medical Radiation Physics, University of Wollongong, Australia \\ ${ }^{2}$ Ionising Radiation, NSTLI Nuclear Stewardship, Australian Nuclear Science and Technology \\ Organisation, Lucas Heights, NSW 2234, Australia \\ ${ }^{3}$ National Institutes for Quantum and Radiological Science and Technology, Chiba, Japan \\ ${ }^{4}$ SINTEF, Norway \\ ${ }^{5}$ University of New South Wales, Sydney NSW 2052, Australia \\ E-mail: db001@uowmail.edu.au
}

\begin{abstract}
Microdosimetry is a particularly powerful method to estimate the relative biological effectiveness (RBE) of any mixed radiation field. This is particularly convenient for therapeutic heavy ion therapy (HIT) beams, referring to ions larger than protons, where the RBE of the beam can vary significantly along the Bragg curve. Additionally, due to the sharp dose gradients at the end of the Bragg peak (BP), or spread out BP, to make accurate measurements and estimations of the biological properties of a beam a high spatial resolution is required, less than a millimetre. This requirement makes silicon microdosimetry particularly attractive due to the thicknesses of the sensitive volumes commonly being $\sim 10 \mu \mathrm{m}$ or less. Monte Carlo (MC) codes are widely used to study the complex mixed HIT radiation field as well as to model the response of novel microdosimeter detectors when irradiated with HIT beams. Therefore it is essential to validate $\mathrm{MC}$ codes against experimental measurements.

This work compares measurements performed with a silicon microdosimeter in monoenergetic ${ }^{12} \mathrm{C},{ }^{14} \mathrm{~N}$ and ${ }^{16} \mathrm{O}$ ion beams of therapeutic energies, against simulation results calculated with the Geant4 toolkit. Experimental and simulation results were compared in terms of microdosimetric spectra (dose lineal energy, $d(y)$ ), the dose mean lineal energy, $y_{D}$ and the $\mathrm{RBE}_{10}$, as estimated by the microdosimetric kinetic model (MKM). Overall Geant4 showed reasonable agreement with experimental measurements. Before the distal edge of the $\mathrm{BP}$, simulation and experiment agreed within $\sim 10 \%$ for $y_{D}$ and $\sim 2 \%$ for $\mathrm{RBE}_{10}$. Downstream of the BP less agreement was observed between simulation and experiment, particularly for the ${ }^{12} \mathrm{C}$ and ${ }^{16} \mathrm{O}$ beams. Simulation results downstream of the BP had lower values of $y_{D}$ and $\mathrm{RBE}_{10}$ compared to the experiment due to a higher contribution from lighter fragments compared to heavier fragments.
\end{abstract}

Keywords: Microdosimetry, Heavy ion therapy (HIT), Silicon-on-insulator (SOI), Geant4, Validation 


\section{Introduction}

Heavy ion therapy (HIT), referring to ions heavier than protons for cancer treatment, has had growing interest in recent years due to its potential for greater dose conformity over both conventional photon X-ray and proton therapy [1]. HIT is also attractive for radioresistant tumours, such as osteosarcomas [2], since HIT does not rely on the production of free radicals nearly as much as conventional radiotherapy. With conventional X-ray radiotherapy having an oxygen enhancement ratio (OER) of $\sim 3$ and ions larger than ${ }^{12} \mathrm{C}$ having an OER of $\sim 1$ [3]. A lower OER has the potential to make tumours with hypoxic regions simpler to treat by not having to know the concentration of oxygen/blood as accurately or account for changes over time. The advantages of a higher LET also brings with it some complications, for therapeutic heavy ions, such as ${ }^{12} \mathrm{C}$, where the LET varies from $\sim 10-1000 \mathrm{keV} \mu \mathrm{m}$ [4], resulting in a relative biological effectiveness (RBE) which varies significantly along the Bragg curve. For successful treatment with HIT it is essential to account for the varying RBE in order to produce a uniform biological dose treatment to the target volume [5].

There are several theoretical methods capable of predicting the changing biological effectiveness in HIT, such as the Local Effect Model (LEM) [6] which uses cell survival data of photons with the track structure of ions. Models such as these calculate the biological effect of the beam theoretically and are not based on making experimental measurements of the ion beam directly. For quality assurance, it is important to be able to directly measure the properties of a delivered beam experimentally and ensure that the properties of the delivered beam match those of the planned beam. One such method to estimate the RBE of an ion beam experimentally, is with microdosimetry and the microdosimetric kinetic model (MKM) [7]. Microdosimetry is the study of energy deposition in micron sized sensitive volumes (SVs) [8], representing the size of cell structures. Microdosimetry measurements are conventionally performed using tissue equivalent proportional counters (TEPCs), with commercial solutions often having a physical SV (not simulated) of the order of $\sim 10 \mathrm{~mm}$ in diameter [9]. These relatively large volume sizes are not ideal for making measurements of the distal edge of a Bragg peak/spread out Bragg peak $(\mathrm{BP} / \mathrm{SOBP})$. Silicon microdosimeters provide a much higher spatial resolution, with SV thicknesses of $\sim 10 \mu \mathrm{m}$ and less commonly being used in hadron therapy beams [10] [11].

Due to the complexity of the radiation field in HIT, with $\sim 70 \%$ of a $400 \mathrm{MeV} / \mathrm{u}{ }^{12} \mathrm{C}$ ion beam undergoing a nuclear reaction before the BP [12], Monte Carlo simulations are often performed to study the radiation field and for comparison against experiment [13]. As such, it is important to know the performance of the physics models used in the Monte Carlo simulation compared to dedicated experimental measurements. This work evaluates the performance of the Monte Carlo toolkit Geant4 [14] [15] [16], for modelling silicon microdosimetry in HIT radiation fields against experimental measurements performed at the Heavy Ion Medical Accelerator in Chiba, (HIMAC), Japan. The studied beams include mono-energetic ${ }^{12} \mathrm{C},{ }^{14} \mathrm{~N}$ and ${ }^{16} \mathrm{O}$ ions. Comparisons between the experiment and simulation are performed in terms of the: microdosimetric spectra, dose mean lineal energy $\left(y_{D}\right)$ and the $\mathrm{RBE}_{10}$, as estimated by the modified microdosimetric kinetic model (MKM) [7].

\section{Materials and Methods}

This work compares experimental measurements obtained using a silicon microdosimeter against simulation results using the Monte Carlo toolkit Geant4, version 10.2p3. The experimental measurements were obtained at the Biological beamline in HIMAC, Japan, which is briefly described in section 2.1. The silicon microdosimeter used was the first generation Mushroom air-trenched microdosimeter, designed by the Centre for Medical Radiation Physics (CMRP) and fabricated by SINTEF [17], and is described in section 2.3. The Mushroom microdosimeter was irradiated with mono-energetic ${ }^{12} \mathrm{C},{ }^{14} \mathrm{~N}$ and ${ }^{16} \mathrm{O}$ ion beams separately, with energies of $290 \mathrm{MeV} / \mathrm{u}, 180 \mathrm{MeV} / \mathrm{u}$ and $400 \mathrm{MeV} / \mathrm{u}$, respectively. The experimental ${ }^{12} \mathrm{C}$ ion measurements 
were originally presented in [10] by Tran et al. and the ${ }^{14} \mathrm{~N}$ and ${ }^{16} \mathrm{O}$ ion results by Tran et al. in [11].

The setup of the experiment and the simulation is shown in figure 1 , which shows the Mushroom microdosimeter placed in a water proof sheath (described in section 2.2), in a water phantom with $6 \mathrm{~mm}$ thick PMMA walls, placed at the iso-centre of the Biological beamline. The water phantom has an area of $220 \times 220 \mathrm{~mm}^{2}$ perpendicular to the beam and a thickness of 260 $\mathrm{mm}$. The microdosimeter was positioned in the centre of the beam (X-Z plane) and was moved through the radiation field in the Y-direction by a motion stage, which allows the position to be placed within an accuracy of a $\mathrm{mm}$.

The electromagnetic interactions were modelled using the G4 Standard EM option 3 physics constructor. The High Precision (HP) Data library was used to model neutron physics processes below $20 \mathrm{MeV}$. The simulations were run two separate times with alternative inelastic hadronic models used to compare to one another. The hadronic interactions of ions were modelled by the G4IonBinaryCascadePhysics (BIC) model and the G4IonQMDPhysics (Quantum Molecular Dynamics-QMD) model. These models were recently validated against experimental measurements of the fragmentation of a ${ }^{12} \mathrm{C}$ ion beam [18].

\subsection{The Biological beamline}

The Biological beamline at HIMAC, shown in figure 1, has been previously modelled in Geant4 and validated for mono-energetic and spread out Bragg peak ${ }^{12} \mathrm{C}$ ion beams by Bolst et al. [19]. It consists of a mono-energetic pencil beam which is shaped by a pair of two wobbling magnets into a circular shape. The lateral dose uniformity of the beam is then improved by passing through scatterers (tantalum in this study) and the neutron contribution in the beam is reduced by a neutron shutter. The beam is shaped by various collimators after the neutron shutter, with the final collimation being performed by a pair of $5 \mathrm{~cm}$ thick brass X-Y collimators. For this study of mono-energetic beams, the ridge filter shown in figure 1 is not used.

The beamline parameters simulated for the three different ion beams considered $\left({ }^{12} \mathrm{C},{ }^{14} \mathrm{~N}\right.$, $\left.{ }^{16} \mathrm{O}\right)$ and their BP position in the water phantom are summarised in table 1 . All beams were collimated to $100 \times 100 \mathrm{~mm}^{2}$ by the X-Y brass collimators just before the phantom. For the ${ }^{12} \mathrm{C}$ ion beam, the magnetic amplitude of the wobbler magnets $\left(B_{0}\right)$ was set to $0.045 \mathrm{~T}$. The ${ }^{14} \mathrm{~N}$ and ${ }^{16} \mathrm{O}$ ion beams, instead of simulating the complete wobbler configuration, used a diverging source, or a "cone" beam, with a divergence of $0.5^{\circ}$. The use of a diverging beam was previously found in [19] to not significantly affect the energy deposition along the centre of the beam, with a difference within $\sim 0.2 \%$ along the entire water phantom. In addition to the increased simplicity of the cone beam, this approach was used due to the lack of lateral dose measurements which are required to finely tune the wobbler strength. The ${ }^{16} \mathrm{O}$ ion beam is the only beam to use PMMA range shifters, with a total thickness of $86 \mathrm{~mm}$ or a water equivalent thickness (WET) of $\sim 100$ $\mathrm{mm}$ and are positioned approximately $300 \mathrm{~mm}$ upstream from the water phantom. The range shifter was used because the beam's range was too large $(\sim 190 \mathrm{~mm})$ to study the BP region in the water phantom. Despite the use of the range shifter, the range of the ${ }^{16} \mathrm{O}$ ion beam are quoted with reference to the depth in the water phantom itself and does not add the additional $100 \mathrm{~mm}$ WET of the range shifter.

\subsection{Water proof sheath and probe}

The water proof PMMA sheath, placed in the water phantom, houses the micro-plus $\left(\mu^{+}\right)$probe, which is an in-house CMRP designed and built probe which provides a compact and low noise readout system. A screenshot of the PMMA probe, modelled in Geant4, is shown in the bottom left diagram of figure 1 . The PMMA sheath is modelled as a $30 \times 30 \times 30 \mathrm{~mm}^{3}$ block covered with a $75 \mu \mathrm{m}$ thick aluminium layer except above the window (see diagram). At the far left of the PMMA sheath, where the beam is incident, there is a $16 \times 16 \mathrm{~mm}^{2}$ cutout which is $4.7 \mathrm{~mm}$ 


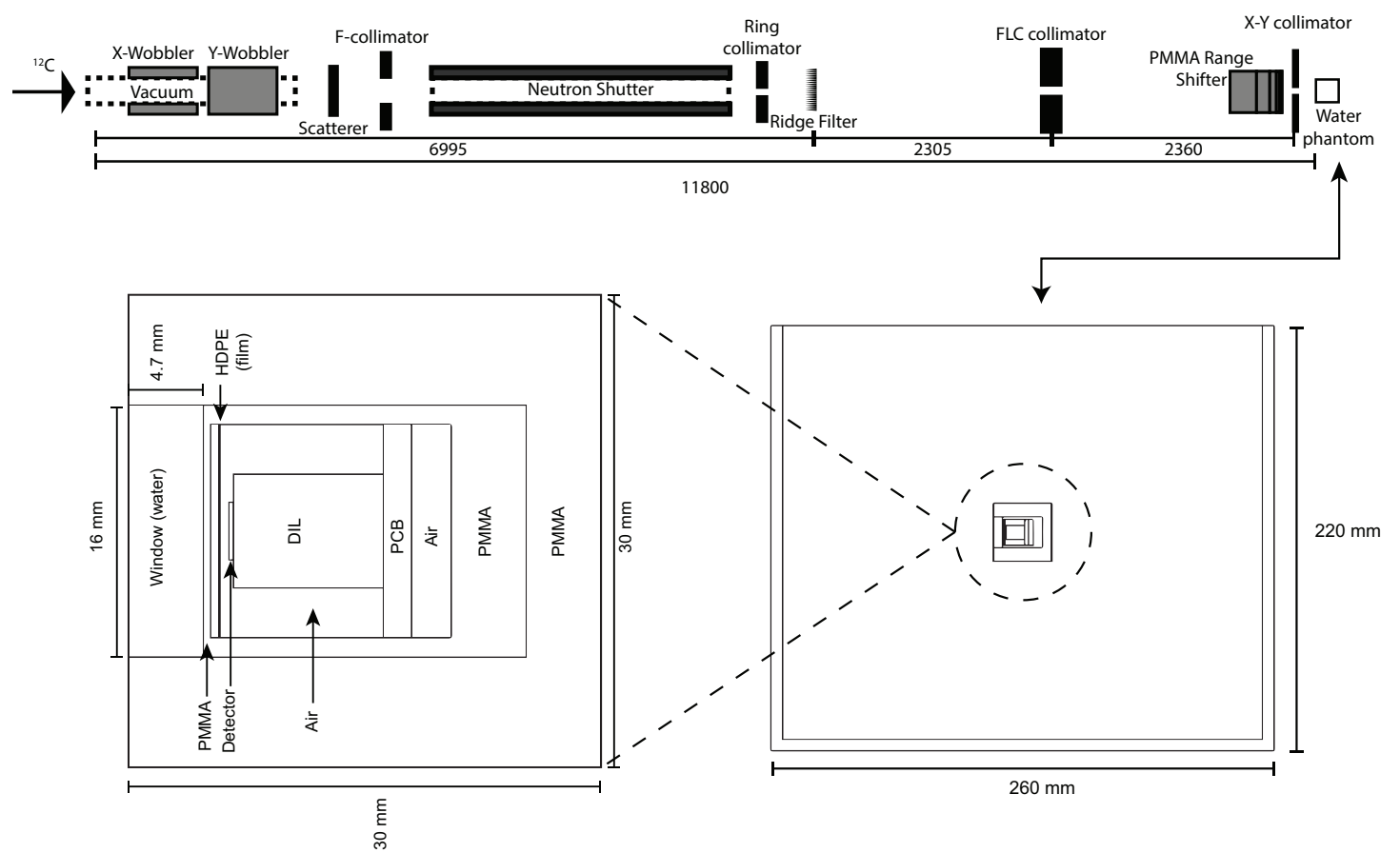

Figure 1: Top: Layout of the Biological beamline modelled in Geant4, not to scale, figure reproduced from [19]. Bottom right: Enlarged view of the water phantom containing the detector probe. Bottom Left: Enlarged view of the water proof probe encasing the detector.

\begin{tabular}{c|ccccc}
\hline Ion & Primary Energy $(\mathrm{MeV} / \mathrm{u})$ & Energy Sigma $(\%)$ & Ta Thickness $(\mathrm{mm})$ & Range shifter $(\mathrm{mm})$ & BP in phantom $(\mathrm{mm})$ \\
\hline${ }^{12} \mathrm{C}$ & 288.6 & 0.2 & 0.434 & 0 & 149 \\
${ }^{14} \mathrm{~N}$ & 180 & 0.36 & 0.434 & 0 & 49 \\
${ }^{16} \mathrm{O}$ & 400 & 0.15 & 0.649 & 86 & $91.5(191.5)$ \\
\hline
\end{tabular}

Table 1: Summary of the configurations of the ion beams and their Bragg Peak (BP) positions considered in this work. The second value of $191.5 \mathrm{~mm}$ for the $\mathrm{BP}$ position in ${ }^{16} \mathrm{O}$ adds the 100 $\mathrm{mm}$ of WET provided by the PMMA range shifter.

deep. This cutout is done to minimise non-water material inline of the beam, with the water proof sheath only adding a $0.5 \mathrm{~mm}$ thickness of PMMA to the beam before the detector. Right of the $0.5 \mathrm{~mm}$ thick PMMA window there is $0.5 \mathrm{~mm}$ of air before a $0.1 \mathrm{~mm}$ thick high density polyethylene (HDPE) film which shields the detector from visible light. Beyond the HDPE film there is another $0.53 \mathrm{~mm}$ of air just before the surface of the Mushroom microdosimeter. The detector is mounted onto an aluminium oxide dual in-line (DIL) package which is on a PCB modelled as pyrex glass.

When measurements are made using the water proof sheath, the reported "depth" is given in terms of the water equivalent thickness (WET) from the face of the water phantom $(6 \mathrm{~mm}$ of PMMA) to the surface of the detector. This involves using conversion factors, calculated using Geant4 by simulating the BP positions for different beam energies and materials. The conversion factor is calculated by taking the ratio of the BP position in water to the other material, which is similar to taking the average ratio of the stopping powers of the two materials. The materials before the detector and their thicknesses, as well as their converted WETs are shown in table 2. 


\begin{tabular}{ccccc}
\hline Item & Material & Thickness $(\mathrm{mm})$ & Conversion factor & WET $(\mathrm{mm})$ \\
\hline Water phantom wall & PMMA & 6 & 1.16 & 6.96 \\
Probe window & PMMA & 0.5 & 1.16 & 0.58 \\
Top air gap & Air & 0.53 & 0.001 & 0.00053 \\
Film & HDPE & 0.1 & 1 & 0.1 \\
Bottom air gap & Air & 0.53 & 0.001 & 0.00053 \\
\hline
\end{tabular}

Table 2: Summary of the different materials, their thicknesses and WETs from the start of the water phantom case to the face of the detector.

\subsection{Air trenched Mushroom microdosimeter}

The Mushroom microdosimeter was modelled in Geant4 based on the images obtained with a scanning electron microscope (SEM) [20]. Diagrams and images of the Mushroom are shown in figure 2, with the top right being an SEM image and the remaining diagrams being screenshots from the Geant 4 simulation. This device is the first generation of its design used by CMRP and exploits 3D technology [21], which has the advantages of: radiation hardness, fast collection times, well defined SVs and low depletion voltages [22].

The Mushroom is fabricated from a high-resistivity p-type silicon of $10 \mathrm{k} \Omega \mathrm{cm}$ and the structural design is refereed to as a trenched 3D. The SV has a trenched hollow core and hollowed semi-circles which extend $\sim 2.7 \mu \mathrm{m}$ from the SV to reduce charge generated outside of the SV from being collected. The SVs cover an area of $\sim 2.5 \times 2.5 \mathrm{~mm}^{2}$ and are connected into arrays of $50 \times 50$ rows and columns by aluminium contacts. The SVs have a thickness of $9.1 \mu \mathrm{m}$ and a diameter of $28.5 \mu \mathrm{m}$. On the top surface of the SV there is a $0.85 \mu \mathrm{m}$ thick overlayer of $\mathrm{SiO}_{2}$ directly on top of the silicon. On the bottom of the silicon SV there is also a 0.85 $\mu \mathrm{m}$ thick $\mathrm{SiO}_{2}$ insulator layer which is above a $300 \mu \mathrm{m}$ thick silicon substrate. The aluminium contacts which connect the SVs together into arrays were modelled as having a width of 3.75 $\mu \mathrm{m}$ and thickness of $1 \mu \mathrm{m}$. More extensive details of the Mushroom microdosimeter as well as experimental and theoretical characterisation of the device can be found in [20].

Every ion beam was measured with a slightly different energy channel calibration. For both the ${ }^{12} \mathrm{C}$ and ${ }^{16} \mathrm{O}$ ion beams, 4096 energy bins were used with a minimum energy deposition of 32.8 and $25.5 \mathrm{keV}$, respectively; while their maximum energy deposition was 22371.6 and 22322.2 $\mathrm{keV}$, respectively. For the ${ }^{14} \mathrm{~N}$ ion beam 8192 energy bins were used instead, with a minimum and maximum energy deposition of 15.8319 and $22314.5 \mathrm{keV}$, respectively. For visual purposes the original linear bins were re-binned into 110 logarithmically spaced bins with a lineal energy range from $0.1-10000 \mathrm{keV} / \mu \mathrm{m}$.

\subsection{Microdosimetric quantities compared between experiment and simulation}

The quantities used to compare simulation and experiment in this work are the dose mean lineal energy, $y_{D}$, and the relative biological effectiveness at $10 \%$ survival, $\mathrm{RBE}_{10}$. The quantities are compared between simulation (Sim) and experiment (Exp) by using the percentage difference, $P D$, as defined in equation 1 . In addition to comparing these two quantities, the microdosimetric spectra are also compared qualitatively in terms of their shape and peak positions.

$$
P D=\left(\frac{E x p-S i m}{E x p}\right) \times 100 \%
$$

Equation 2 is used to calculate the lineal energy, $y$, from the energy deposition, $E$, in the the Mushroom microdosimeter. The $\kappa$ coefficient converts the energy deposited in the silicon SV to a material representing a tissue equivalent medium (striated muscle in this work). In this work $\kappa$ is fixed to 0.57 based on the work by [23]. The lineal energy is calculated by using the mean 


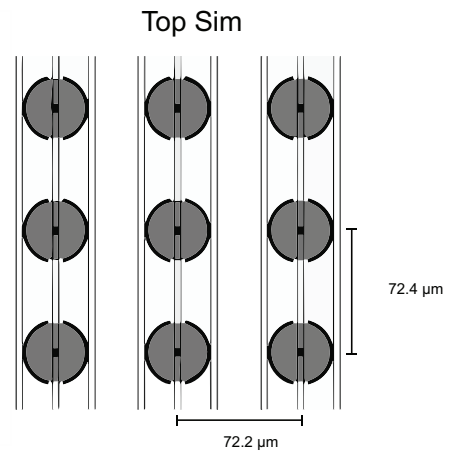

Top Sim

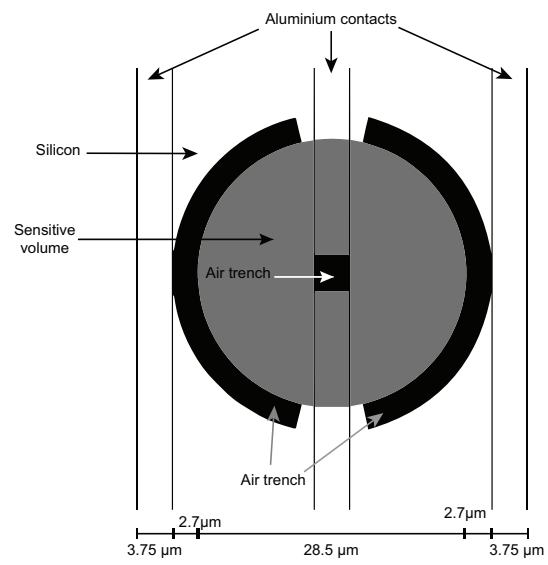

Isometric SEM

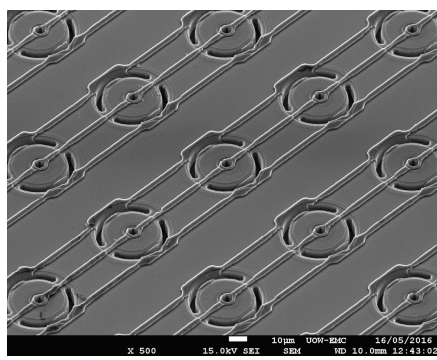

Side Sim

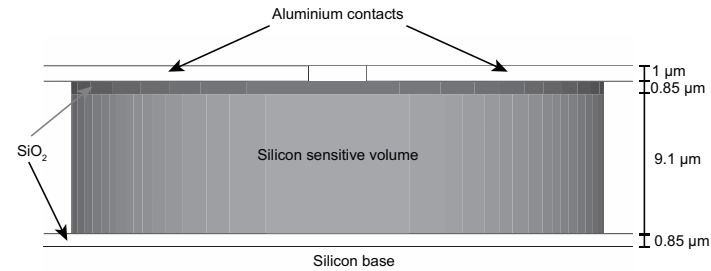

Figure 2: The Mushroom microdosimeter modelled in Geant4 and an SEM image (top right) of the real world device.

path length of the silicon SV $\left(\left\langle l_{\text {Path,Si }}\right\rangle\right)$ instead of the traditional mean chord length calculated using Cauchy's equation, which is valid only for isotropic fields. The mean path length was demonstrated to be more appropriate in [23] to calculate the lineal energy in HIT beams due to their high directionality of HIT beams. Additionally, for simplicity the mean path length is fixed in this study, equal to the thickness of the SV, $9.1 \mu \mathrm{m}$, based on [23].

The probability distribution of the lineal energy (equation 2) is denoted as $f(y)$. The dose mean lineal energy, $y_{D}$, is the ratio of the second and first moments of $f(y)$ and is shown in equation 3 .

$$
\begin{gathered}
y=\frac{\kappa E}{\left\langle l_{\text {Path }, S i}\right\rangle} \\
y_{D}=\frac{\int y^{2} f(y) d y}{\int y f(y) d y}
\end{gathered}
$$

The $\mathrm{RBE}_{10}$ at different positions in the beam was estimated using the modified MKM, which uses the measured microdosimetric spectrum $(f(y))$ as input to the model. The MKM was originally formulated by Hawkins in 1994 [24] and is based on dividing the cell nucleus into subnuclear sites called "domains" and assumes that the response of these domains from radiation follows a linear quadratic curve. The MKM was later modified by Kase et al. [7] to account for 
over-killing effects, which is important for HIT. The output from the MKM is an estimated cell survival response from radiation $\left(\mathrm{RBE}_{10}\right)$ for a particular cell. In order to make this prediction, certain cell specific parameters must be measured such as the parameters of the linear-quadratic model of cell survival, $\alpha_{0}$ and $\beta$ for photon irradiation. The $\alpha_{0}$ and $\beta$ parameters describe the response of cells when irradiated with different amounts of dose, with $\alpha_{0}$ describing the initial response of cells at lower doses and $\beta$ describing the response at higher doses [25]. In addition to $\alpha_{0}$ and $\beta$, the domain "size" $\left(r_{d}\right)$ and the LET where over-killing begins for the cell type are also required in order to predict the cells' response. In this work, the estimated $\mathrm{RBE}_{10}$ is calculated for human salivary glands (HSG) cells, using cell parameters measured by Kase et al. [7]. The $\alpha_{0}$ and $\beta$ values measured for HSG cells were $0.13 \mathrm{~Gy}^{-1}, 0.05 \mathrm{~Gy}^{-2}$, respectively, while the domain radius had a value of $0.42 \mu \mathrm{m}$ with an assumed density of tissue of $1 \mathrm{~g} / \mathrm{cm}^{3}$ ( $\rho$ in the equation below). Finally, the LET of radiation where over-killing effects begins was determined to be $150 \mathrm{keV} / \mu \mathrm{m}$ and is called the saturation parameter and labelled as $y_{0}$ in the modified MKM. Once $y_{0}$ is determined for a particular cell the saturation-corrected dose mean lineal energy, $y^{*}$, can be calculated using the measured microdosimetric spectrum and equation 4. From $y^{*}$ the $\alpha$ value can be calculated from equation 5 and then the estimated $\mathrm{RBE}_{10}$ can be calculated using equation 6 , where $D_{10, x-\text { ray }}$ is the dose needed to obtain a $10 \%$ cell survival of HSG cells for a $200 \mathrm{kVp}$ x-ray source and was measured by Kase et al. to be $10 \mathrm{~Gy}$.

$$
\begin{gathered}
y^{*}=y_{0}^{2} \frac{\int\left(1-\exp \left(-y^{2} / y_{0}^{2}\right)\right) f(y) d y}{\int y f(y) d y} \\
\alpha=\alpha_{0}+y^{*} \frac{\beta}{\rho \pi r_{d}^{2}} \\
R B E_{10}=\frac{2 \beta D_{10, x-r a y}}{\sqrt{\alpha^{2}-4 \beta \ln (0.1)}-\alpha}
\end{gathered}
$$

In order to obtain sufficient statistics, in the form of "hits" or counts in the microdosimeter, the number of primary ion particles generated in the simulation was varied depending on where along the beam the microdosimeter was positioned. For positions upstream of the BP the number of primary ions generated varied between $\sim 1$ and $2 \times 10^{7}$, while for positions at the pinnacle and distal edge of the BP $2 \times 10^{7}$ primary ions were generated. Due to the loss of the primary beam, positions downstream of the BP had $\sim 10^{8}$ primary ions generated. The error bars plotted in this work only reflect the statistical error in the number of counts in the channels of the detector and do not consider any other uncertainties, such as cell specific parameters for the estimated $\mathrm{RBE}_{10}$. The uncertainty of the simulation is calculated by splitting the total number of counts recorded in the detector into ten groups and taking the standard deviation of the quantity of interest $\left(y_{D}\right.$ or $\left.\mathrm{RBE}_{10}\right)$ and is plotted with a $68 \%$ confidence limit. The error bars associated with the experiments are based on error propagation, with the starting error being the square root of the counts in each bin. The error bars displayed on the microdosimetric plots are calculated the same way for both the experiment and the simulation and are based on the number of counts in each logarithmically spaced bin.

\section{Results}

For the results presented below the comparisons between simulation and experiment are primarily with respect to the BIC model, with discussion of QMD results left for the Discussion section (section 4). The microdosimetric spectra (figures 3,5 and 8) shown below are all generated with the BIC model, where "Total" and each particle's contribution to the spectrum refers to the BIC model's response. However, for these three figures there is one plot corresponding to $5 \mathrm{~mm}$ downstream of each beam's BP, where "Total" and each particle's 
spectra for each particle refers to the QMD model, with the total BIC response also plotted for reference.

\subsection{Mono-energetic $290 \mathrm{MeV} /{ }^{12} \mathrm{C}$ ion beam}

The dose weighted microdosimetric spectra, displayed on the traditional semi-log scale, $y d(y)$, at various depths in the water phantom along the mono-energetic ${ }^{12} \mathrm{C}$ ion beam and downstream of the BP, are shown figure 3 . The top left plot shows the microdosimetric spectra at the entrance of the phantom $(18.91 \mathrm{~mm})$ while $149 \mathrm{~mm}$ corresponds to the pinnacle of the BP. The contribution to the total dose deposited in the detector by different particle types in the simulation is also plotted. The ${ }^{12} \mathrm{C}$ ion peak (dashed green line) on the right of the spectra is observed to dominate the spectra, with secondary fragments only making noticeable contributions at the distal part of the BP and beyond, as expected.

From the entrance of the phantom to the pinnacle of the BP, the lineal energy of the ${ }^{12} \mathrm{C}$ peak increases from $\sim 10$ to $\sim 100 \mathrm{keV} / \mu \mathrm{m}$, with a stopper peak at the far right of the spectrum with a lineal energy of $\sim 700 \mathrm{keV} / \mu \mathrm{m}$. At the end of an ion's range, its LET increases rapidly, meaning that a small change in depth of a few 100 microns can drastically change the value of $y_{D}$. This rapid change in LET, together with the sharp dose gradients at the end of an ion's range, means that with the current positional uncertainty in the water phantom of $\sim 1 \mathrm{~mm}$, at the distal edge of the BP the agreement between experiment and simulation is harder to achieve.

After the incident beam has stopped, the microdosimetric spectrum is significantly different as it is produced by the secondary mixed radiation field only. The lineal energy peaks of the microdosimetric spectrum at $155 \mathrm{~mm}$ (6 $\mathrm{mm}$ distal from the BP pinnacle) can be seen to align reasonably well when comparing simulation results and experimental measurements, with the most prominent peak being mostly from boron fragments. However, at the lower lineal energies the simulation is seen to have an excessive proportion of lighter fragments compared to larger fragments, with ${ }^{4} \mathrm{He}$ being very dominant in the simulation.

The calculated values of $y_{D}$ and $\mathrm{RBE}_{10}$, using the MKM, are shown in figure 4 . The right plots show an enlarged view of the BP region of the left plots. For reference, the energy deposition scored in $0.1 \mathrm{~mm}$ thick water slabs along the phantom by the simulation (without the detector/probe present) is plotted in red.

Comparing the $y_{D}$ values of experiment and simulation, excellent agreement is observed for both the values and the shape, particularly at the BP. Upstream of the BP region, the $y_{D}$ is seen to be fairly constant with a value of $\sim 20 \mathrm{keV} / \mu \mathrm{m}$ reaching a maximum of $250 \mathrm{keV} / \mu \mathrm{m}$ (simulation) to $300 \mathrm{keV} / \mu \mathrm{m}$ (experiment). It can be seen that for a few depths upstream of the $\mathrm{BP}$, particularly at $18.91 \mathrm{~mm}$, that the error bars of the simulation are quite large compared to other depths. This large error bar occurs due to a few high LET particles depositing energy in the SV. Downstream of the BP the simulation is consistently lower than the experiment. Which as discussed with the microdosimetric spectra in figure 3, the simulation is characterised by an overproduction of lighter fragments (mostly likely He fragments) with respect to heavier fragments.

For $\mathrm{RBE}_{10}$ values the agreement is slightly better than the $y_{D}$ results, with the difference being $\sim 2 \%$ before the distal part of the $\mathrm{BP}$. The $\mathrm{RBE}_{10}$ has noticeably less fluctuations than $y_{D}$, this can be attributed to $y_{D}$ being proportional to $y^{2}$ while $\mathrm{RBE}_{10}$ is proportional to $\left(1-\exp \left(-y^{2} / y_{0}^{2}\right)\right)$. This causes infrequent, high $y$ events, to have a much greater influence on $y_{D}$ compared to $\mathrm{RBE}_{10}$, particularly when $y$ is over $150 \mathrm{keV} / \mu \mathrm{m}$ (the saturation parameter value used by the modified MKM). At the entrance of the phantom the $\mathrm{RBE}_{10}$ is $\sim 1.17$ and reaches a maximum at the pinnacle of the $\mathrm{BP}$ with a value of $\sim 2.9$. 

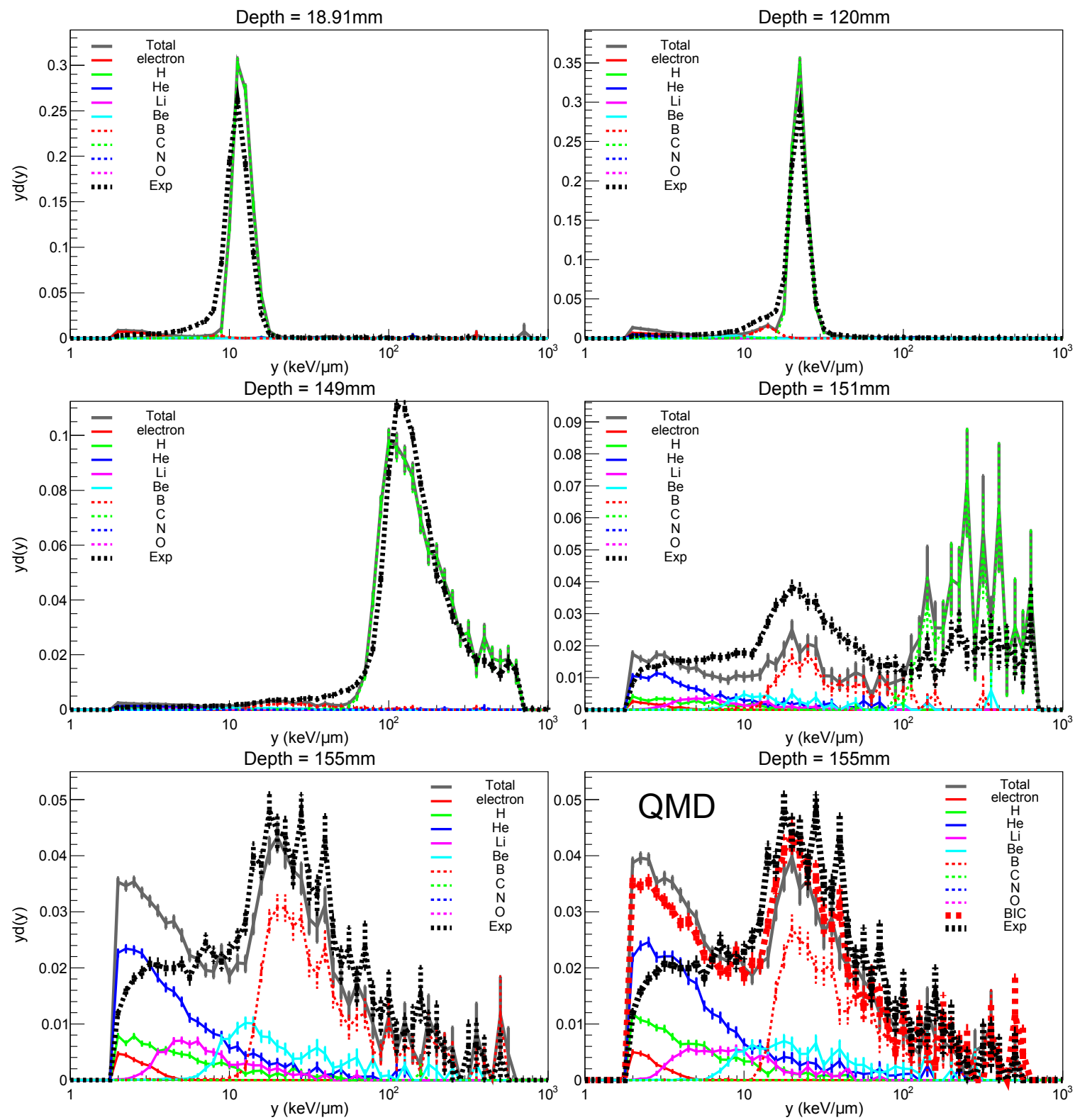

Figure 3: Comparison between the microdosimetric spectra calculated with Geant4 (continuous line) and the experimental measurements (dashed line) at different depths along a $290 \mathrm{MeV} / \mathrm{u}$ ${ }^{12} \mathrm{C}$ ion beam in a water phantom. All simulation spectra shown are obtained with the BIC model except for the $155 \mathrm{~mm}$ depth labelled with "QMD", which was calculated with the QMD model. 

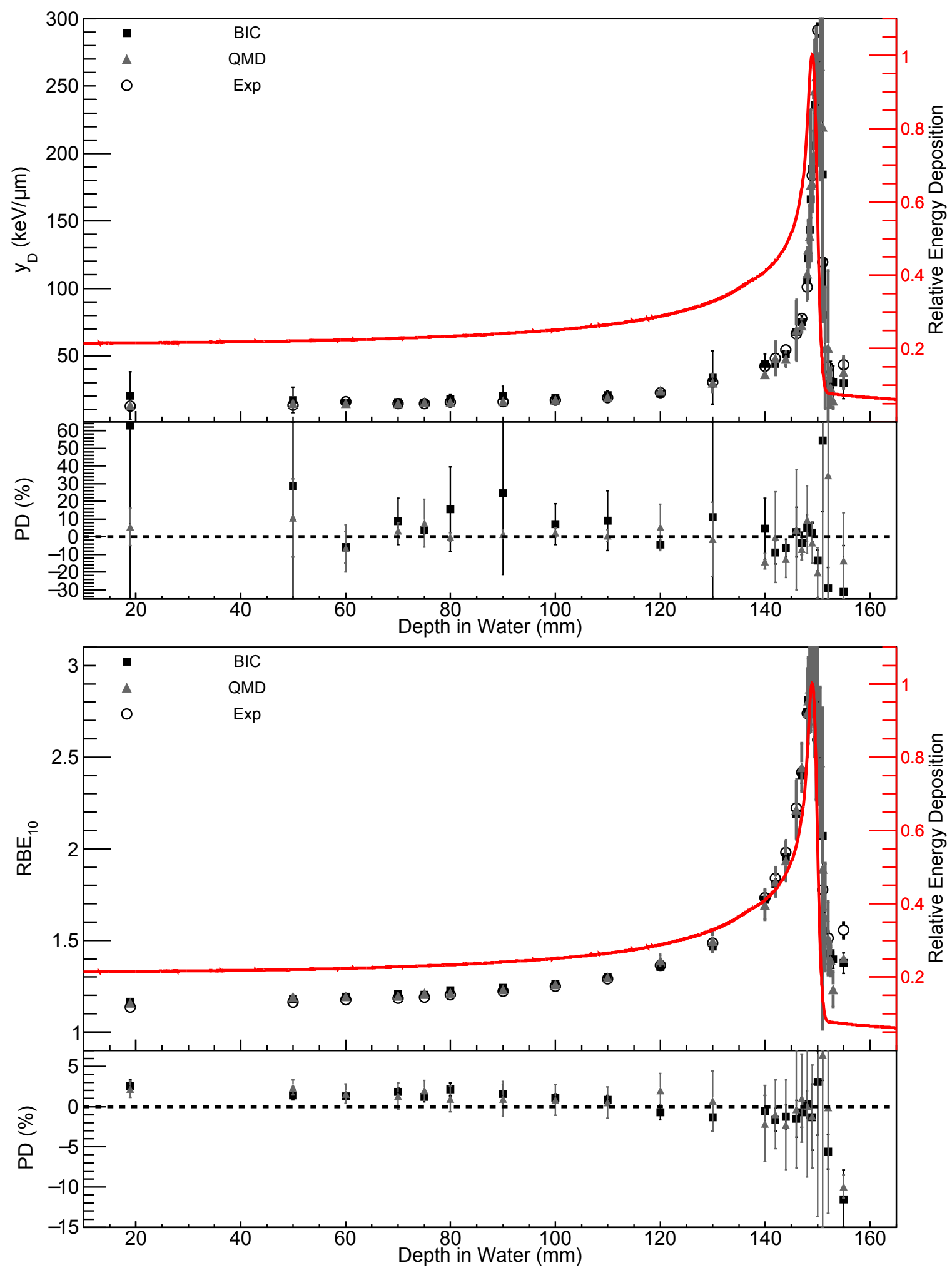

Figure 4: Calculated $y_{D}$ (top) and $\mathrm{RBE}_{10}$ (bottom) values in the $290 \mathrm{MeV} / \mathrm{u}{ }^{12} \mathrm{C}$ ion beam using both the measured and simulated spectra from the Mushroom microdosimeter. The lower panels in both graphs show the percentage deviation (PD) between both data sets. 


\subsection{Mono-energetic $180 \mathrm{MeV} / \mathrm{u}^{14} \mathrm{~N}$ ion beam}

A selection of microdosimetric spectra at different depths in the water phantom (upstream and downstream of the BP), when irradiated with a mono-energetic $180 \mathrm{MeV} / \mathrm{u}^{14} \mathrm{~N}$ ion beam is shown in figure 5 . The stopper peak can be seen to occur at a lineal energy of $\sim 900 \mathrm{keV} / \mu \mathrm{m}$. For the downstream depths, better agreement is observed between simulation and experiment compared to the case of the ${ }^{12} \mathrm{C}$ ion beam. This can be attributed to the smaller range of the ${ }^{14} \mathrm{~N}$ beam, which is $100 \mathrm{~mm}$ less than the ${ }^{12} \mathrm{C}$ beam, leading to a reduced fragment build up curve and causing heavier fragments, such as boron, to have a much shorter range after the BP. The fragment energy peaks of the simulation and experiment again align well with one another and beyond $55 \mathrm{~mm}{ }^{4} \mathrm{He}$ is the dominant fragment for both simulation and experiment.

A comparison between the experimental and simulated $y_{D}$ and $\mathrm{RBE}_{10}$ are shown in figure 6. Good agreement is again seen between experiment and simulation, with downstream of the $\mathrm{BP}$ having much better agreement compared to ${ }^{12} \mathrm{C}$ due to the reduced contribution from larger fragments. The agreement between experiment and simulation is similar to the ${ }^{12} \mathrm{C}$ ion beam case, with agreement being $\sim 10 \%$ for $y_{D}$ before the distal $\mathrm{BP}$ and $\sim 2 \%$ for $\mathrm{RBE}_{10}$. The $y_{D}$ has a value of $\sim 28 \mathrm{keV} / \mu \mathrm{m}$ at the entrance of the phantom and reaches a value of $\sim 400 \mathrm{keV} / \mu \mathrm{m}$ at the distal edge of the $\mathrm{BP}$ at $\sim 50.5 \mathrm{~mm}$ depth. The $\mathrm{RBE}_{10}$ has a value of $\sim 1.5$ at the entrance of the phantom and reaches a maximum of $\sim 3.1$ just before the pinnacle of the BP. The $\mathrm{RBE}_{10}$ is seen to peak before the $\mathrm{BP}$ compared to the ${ }^{12} \mathrm{C}$ ion beam which peaks at the $\mathrm{BP}$ itself due to the higher LET of the ${ }^{14} \mathrm{~N}$ ion beam causing over-killing effects to occur earlier along the Bragg curve.

\subsection{Mono-energetic $400 \mathrm{MeV} / \mathrm{u}^{16} \mathrm{O}$ ion beam}

The microdosimetric spectra in the case of a mono-energetic ${ }^{16} \mathrm{O}$ ion beam for various depths in the phantom are shown in figure 7. It should be re-iterated here that the ${ }^{16} \mathrm{O}$ ion beam had a $100 \mathrm{~mm}$ WET (corresponding to $86 \mathrm{~mm}$ of PMMA) placed approximately $300 \mathrm{~mm}$ before the phantom to shift the location of the BP in the water phantom. Despite the use of the range shifter the depths quoted here are with respect to the detector's position inside the water phantom. Comparing the microdosimetric spectra upstream of the BP, experiment and simulation agree well in terms of position and shape of the incident ${ }^{16} \mathrm{O}$ ion beam, with the stopper peak increasing to a higher lineal energy of $\sim 1000 \mathrm{keV} / \mu \mathrm{m}$. Due to the larger range of the ${ }^{16} \mathrm{O}$ ion beam, the downstream microdosimetric spectra present a more similar trend to the ${ }^{12} \mathrm{C}$ ion beam than the ${ }^{14} \mathrm{~N}$ ion beam, with a more noticeable over contribution of lighter fragments (with He fragments again being the most represented in the simulation).

The $y_{D}$ and $\mathrm{RBE}_{10}$ values along the depth of the phantom are shown in figure 8 . As with the previous beams, $y_{D}$ gives an agreement of $\sim 10 \%$ and $\mathrm{RBE}_{10} \sim 2 \%$, both before the distal edge of the $\mathrm{BP}$, after the BP the discrepancy becomes larger due to the accuracy of the fragmentation model used (BIC). The $y_{D}$ has a value of $\sim 22 \mathrm{keV} / \mu \mathrm{m}$ at the entrance of the phantom and increases to a value of $\sim 320 \mathrm{keV} / \mu \mathrm{m}$ just at the start of the distal edge of the BP at $\sim 93 \mathrm{~mm}$. The $\mathrm{RBE}_{10}$ has a value of 1.35 at the entrance of the water phantom and reaches a maximum of 2.9 just before the pinnacle of the $\mathrm{BP}$ due to over-killing. The values of $y_{D}$ and $\mathrm{RBE}_{10}$, at the surface of the phantom, for ${ }^{16} \mathrm{O}$ ions are slightly lower than ${ }^{14} \mathrm{~N}$ ions. This is due to the much smaller range of ${ }^{14} \mathrm{~N}$ causing less straggling to occur as well as a smaller contribution from fragments due to a reduced fragment build up curve. 

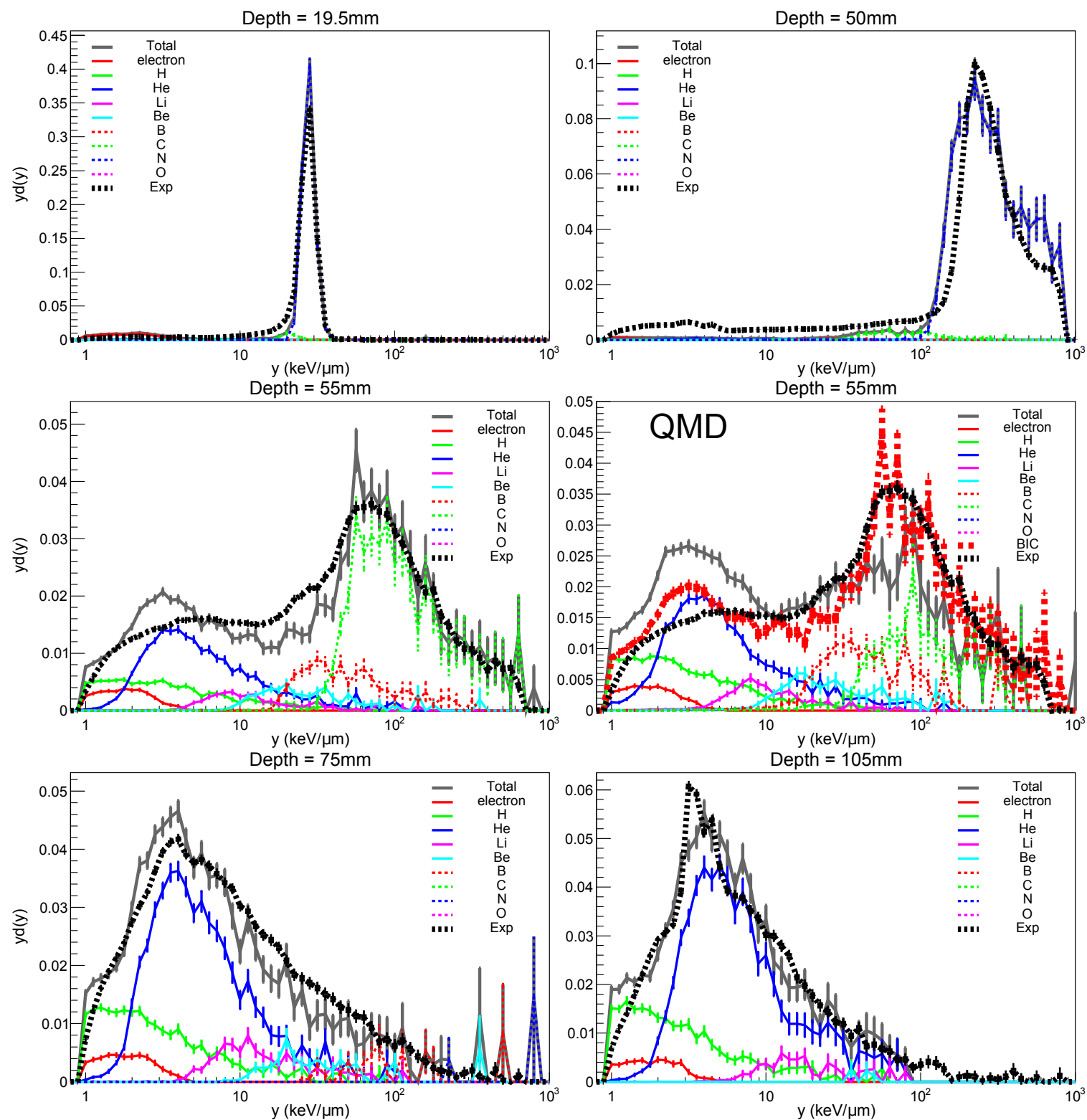

Figure 5: Comparison between the microdosimetric spectra calculated with Geant4 (continuous line) and the experimental measurements (dashed line) at different depths along a $180 \mathrm{MeV} / \mathrm{u}$ ${ }^{14} \mathrm{~N}$ ion beam in a water phantom. All simulation spectra shown are obtained with the BIC model except for the $55 \mathrm{~mm}$ depth labelled with "QMD", which was calculated with the QMD model. 

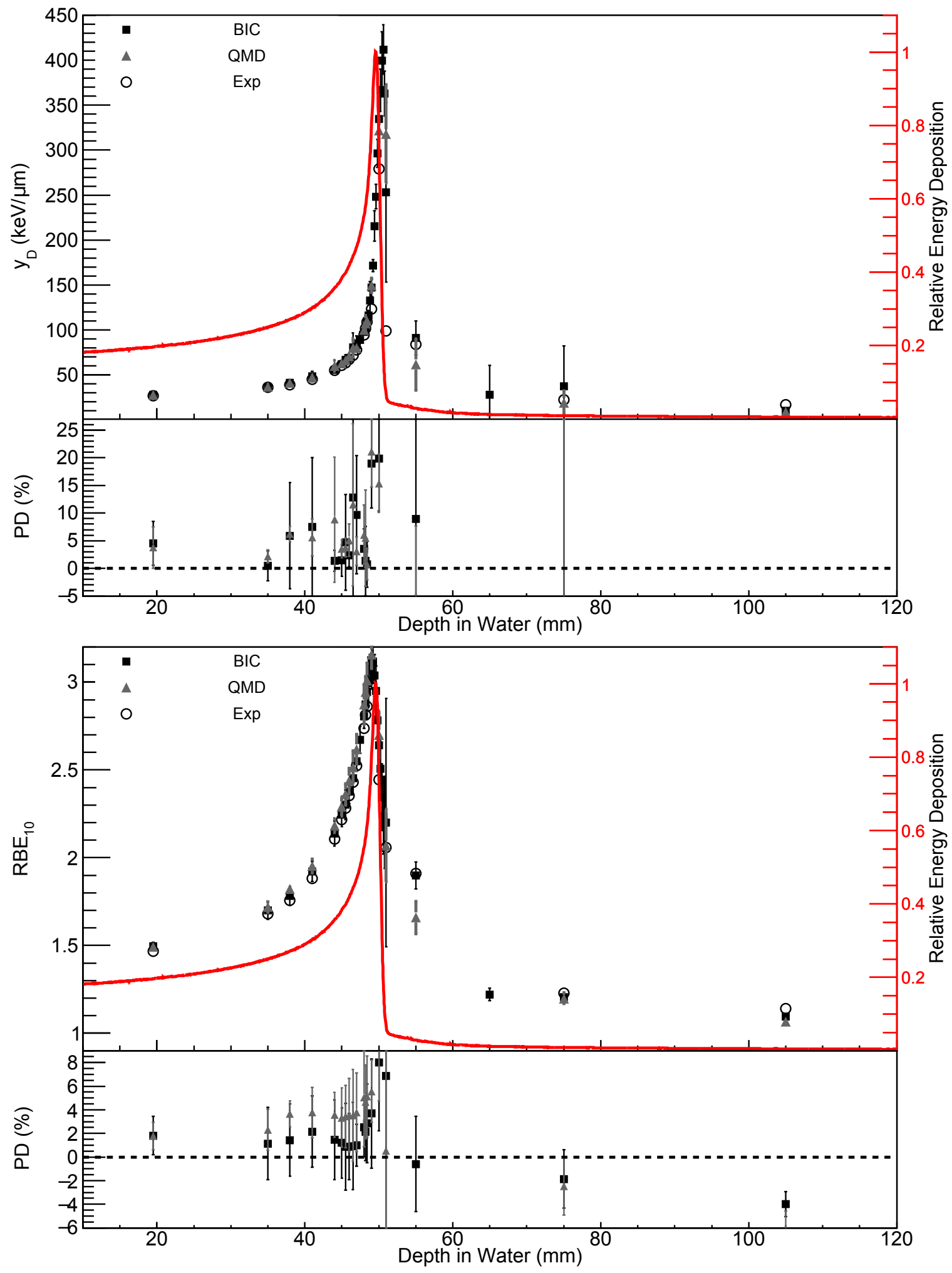

Figure 6: $y_{D}$ (top) and $\mathrm{RBE}_{10}$ (bottom) values values in the $180 \mathrm{MeV} / \mathrm{u}{ }^{14} \mathrm{~N}$ beam using both the measured and simulated spectra from the Mushroom microdosimeter. The lower panels in both graphs show the percentage deviation $(\mathrm{PD})$ between both data sets. 

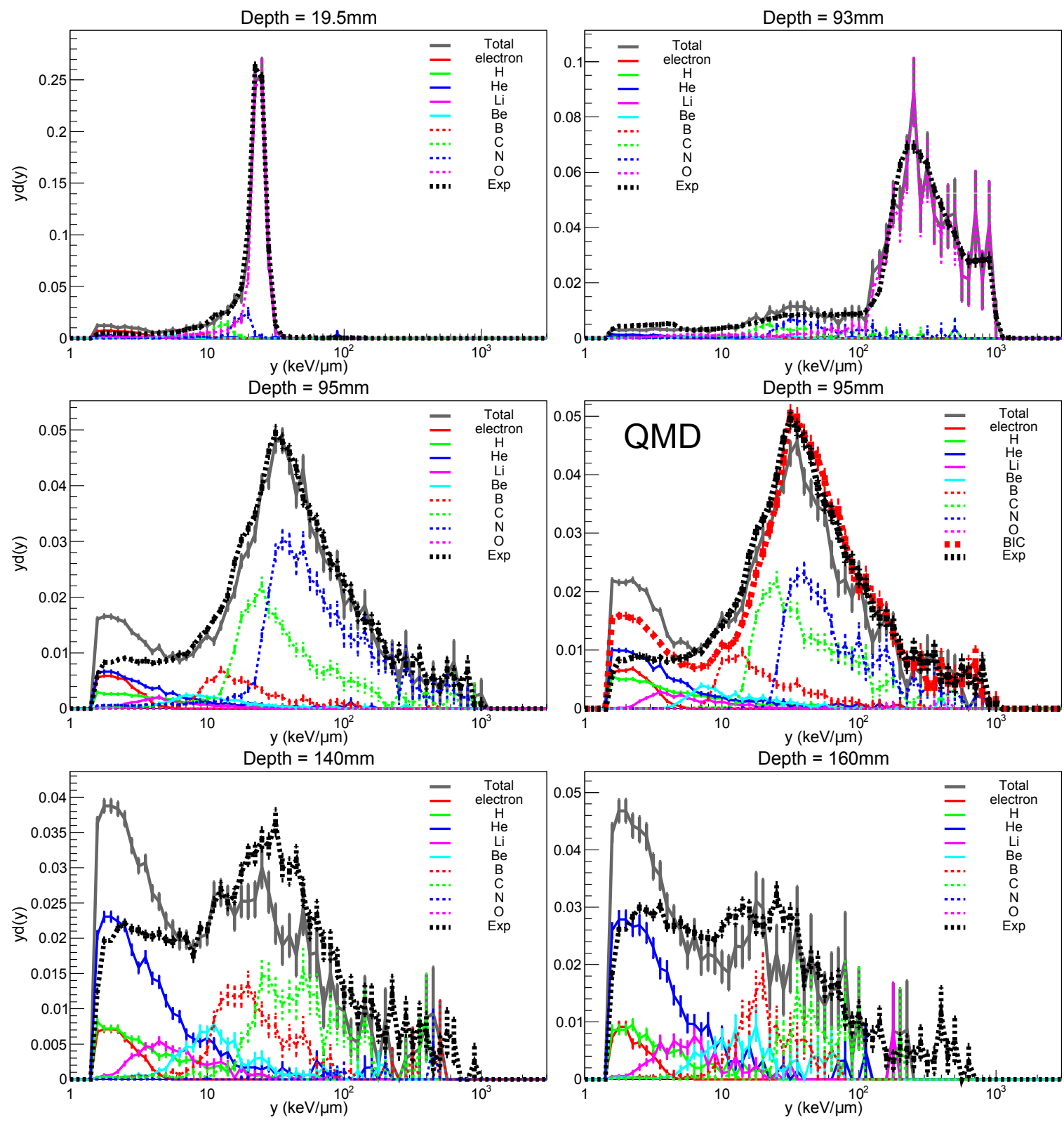

Figure 7: Comparison between the microdosimetric spectra of the simulated Mushroom detector and the experimental device when irradiated in a mono-energetic $400 \mathrm{MeV} / \mathrm{u}^{16} \mathrm{O}$ beam at various depths along the centre of the beam. All spectra are obtained with the BIC model except for the $95 \mathrm{~mm}$ depth on the right side which shows the response from QMD. 

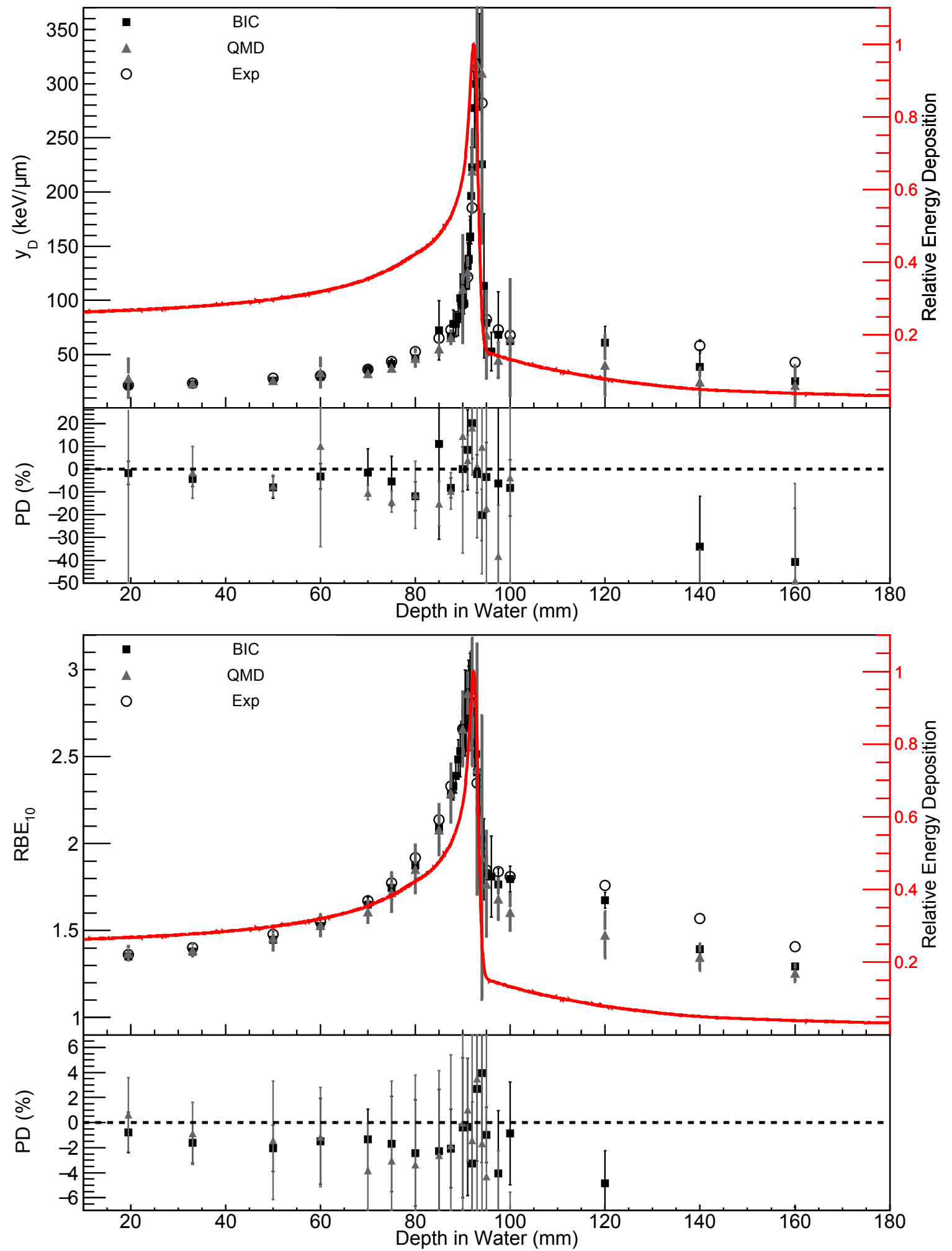

Figure 8: Calculated $y_{D}$ (top) and $\mathrm{RBE}_{10}$ (bottom) values in the $400 \mathrm{MeV} / \mathrm{u}^{16} \mathrm{O}$ beam using both the measured and simulated spectra from the Mushroom microdosimeter. The lower panels in both graphs show the percentage deviation (PD) between both data sets. 


\section{Discussion}

As the incident primary ion traverses the target it will be attenuated based on its total reaction cross-section. As the primary ion beam attenuates it will produce different fragments based on the charge and mass changing cross-sections of the ion. The total reaction cross section of different projectiles and targets are well described in Geant4 and agree well with experimental measurements. The production cross sections of fragments are much more complex to describe due to not only needing to describe their production but also their angular and energy distributions. The ability of Geant 4 to describe experimental measurements of the attenuation of a beam versus the production of fragments can be seen online (https://geant-val.cern.ch/), in particular the Charge Changing Cross-Section test (CCCStest), which compares the total and charge changing cross-sections in Geant 4 for a ${ }^{12} \mathrm{C}$ ion beam. Additionally, the Hadron Data test (TestHD) compares the total reaction cross-section for various projectiles/targets and energies.

Due to the higher lineal energy of the incident primary beam compared to secondary fragments and its attenuation being fairly well described, the agreement between experiment and simulation is fairly consistent for all beams before the BP, with an agreement between $\sim 10 \%$ for $y_{D}$ and $\sim 2 \%$ for $\mathrm{RBE}_{10}$. As mentioned earlier, the $\mathrm{RBE}_{10}$ has noticeably less fluctuations than $y_{D}$ due to their different dependence of the lineal energy spectra.

The observed agreement upstream reflects the dominance of the primary incident beam on the microdosimeter response, while the agreement downstream of the BP is dependent upon the model used and the fragments it generates. The alignment of the peaks of the microdosimetric spectra suggest that the model is adequate at generating the energy distribution of fragments. However, some discrepancies of the primary beam shape are seen between experiment and simulation, particularly on the left side of the peak. This is largely due to the charge collection of the device, where regions of the microdosimeter have a smaller charge collection efficiency compared to the majority of the SV region; charge collection maps of this device can be found in [20]. The effect of taking into account the charge collection distribution of the device in the simulation was investigated in [26] (chapter 6). It was found that the shape of the primary peak of the simulation was improved when applying the charge collection of the device but the spectra of the secondary fragments was less significantly impacted due to their large energy distribution compared to the primary ion beam.

Downstream of the $\mathrm{BP}$ for the ${ }^{12} \mathrm{C}$ and ${ }^{16} \mathrm{O}$ ion beams, which have a significantly larger range than ${ }^{14} \mathrm{~N}$ which causes a greater fragment build-up as mentioned earlier, it was observed that there were more lighter fragments present in the simulation than experiment. This trend mimics the fragment study of [18], which compared the fragment distributions between experimental data [12] against Geant4 simulations for a $400 \mathrm{MeV} / \mathrm{u}^{12} \mathrm{C}$ ion beam incident upon a water target. The fragment study showed that, in general, larger fragments gave less agreement than lighter ones, in terms of the angular distribution. With larger fragments in the Geant4 simulation producing broader angular distributions compared to experimental measurements, this causes a greater proportion of larger fragments to leave the central beam axis compared to lighter fragments in the simulation. This effect is amplified further downstream of the BP, which is seen the most in this current work for the ${ }^{16} \mathrm{O}$ ion beam at $140 \mathrm{~mm}$ to $160 \mathrm{~mm}$, where the disagreement increases.

Due to the dominance of the primary beam, the results do not differ significantly between BIC and QMD upstream of the BP, both in terms of the microdosimetric spectra and the $y_{D}$ and $\mathrm{RBE}_{10}$ quantities. Downstream of the $\mathrm{BP}, \mathrm{QMD}$ shows a greater contribution from lighter ions and BIC has a greater contribution of fragments with a charge of 1 less than the primary ion (Boron for ${ }^{12} \mathrm{C}$, Carbon for ${ }^{14} \mathrm{~N}$ and Nitrogen for ${ }^{16} \mathrm{O}$ ). This trend was also observed in previous work [18], with QMD having a broader angular distribution of fragments compared to BIC. This results in QMD having slightly smaller values than BIC for $y_{D}$ and $\mathrm{RBE}_{10}$, as is most readily seen for ${ }^{16} \mathrm{O}$ which has the largest primary ion range. The lower values obtained 
with the QMD models result in larger differences between experiment compared to the BIC model. An important consideration when choosing a model for Monte Carlo simulations is the computational resources required. As reported previously in [18], the runtime of QMD for thick targets was $\sim 2-8$ times longer than the BIC model, for this study the observed increase in runtime for most depths was mostly between 2-3 times longer. Due to this significant increase in runtime for very little difference in results, particularly for positions where the primary ions reach the detector, it can be difficult to justify the use of the QMD model over the BIC model for the configuration studied here.

The agreement between experiment and simulation for values of $y_{D}$ are similar to those found in a study performed by Böhlen et al. [27], which compared the experimental microdosimetric spectra of ${ }^{12} \mathrm{C}$ ion beams, measured using TEPCs, against simulation results of the FLUKA code [28]. One of the experiments used to compare values of $y_{D}$ against simulation was the work of Martino et al. [29]. Agreement between the experimental measurements and FLUKA in the work of Böhlen was within $\sim 4 \%$ for $y_{D}$ values for measurements at the centre of the beam while those made $20 \mathrm{~mm}$ from the centre of the beam were within $11 \%$. One notable difference between the setup compared by Böhlen et al. and this study is that the beam size of $\sim 2-3$ $\mathrm{mm}$ in diameter, and the size of the TEPC's SV chamber used was $12.7 \mathrm{~mm}$ in diameter. This contrasts with the $100 \mathrm{~mm}$ diameter beam in this study and the $2500 \mathrm{SVs}$ placed over an area of $\sim 13 \mathrm{~mm}^{2}$. The much larger beam size in this study may cause an increased contribution in the detector response from fragments being directed outward from the primary path with fragments generated laterally from the centre of the beam reaching the detector in the centre. Additionally, the previous work used an active beam versus the passive beamline used in this study. This means that the mixed radiation fields may have significant differences in terms of fragment contribution.

\section{Conclusion}

This work evaluated the performance of Geant4 to simulate the response of silicon microdosimeters when irradiated with therapeutic ${ }^{12} \mathrm{C},{ }^{14} \mathrm{~N}$ and ${ }^{16} \mathrm{O}$ ion beams. Simulation results were benchmarked against published experimental measurements performed at the HIMAC facility in Japan. Using a previously validated model of the HIMAC Biological beamline, the silicon microdosimeter used in previous published experiments was accurately modelled and irradiated using the beamline model.

The dose mean lineal energy $\left(y_{D}\right)$ and the $\mathrm{RBE}_{10}$, as estimated by the MKM, of each ion beam was compared between simulation and experiment from upstream of the Bragg curve to downstream of the fragment tail. Experimental measurements and simulation results agree reasonably well along the primary ion beam. Due to the dominance of the primary ion beams on the microdosimetric spectra, the agreement between experiment and simulation stayed fairly constant before the BP with an agreement within $\sim 10 \%$ for $y_{D}$ and $\sim 2 \%$ for $\mathrm{RBE}_{10}$.

Downstream of the BP there was less agreement observed between simulation and experiment, with the simulation having lower values of $y_{D}$ and $\mathrm{RBE}_{10}$ due to the higher representation of lighter fragments compared to heavier fragments. However, the ${ }^{14} \mathrm{~N}$ beam gave fairly good agreement downstream of the $\mathrm{BP}$ due to the smaller range of the beam causing lighter fragments to dominate. Despite the contribution of fragments not giving the best agreement between experiment and simulation, the lineal energy peaks of the fragment distributions did give good agreement. The trends of fragments seen between simulation and experiment from the Mushroom microdosimeter agree with the results from previous work, where angular distributions of fragments were compared to experiment and showed larger fragments having broader angular distributions.

These results demonstrate that Geant 4 is suitable for the simulation of silicon microdosimetry in heavy ion therapy within $\sim 10 \%$ for $y_{D}$ and $\sim 2 \%$ for $\mathrm{RBE}_{10}$, as estimated by the MKM. 


\section{Acknowledgement}

This work was supported by computational resources provided by the Australian Government through the Raijin cluster under the National Computational Merit Allocation Scheme.

\section{References}

[1] T. Ohno, "Particle radiotherapy with carbon ion beams," EPMA Journal, vol. 4, no. 1, p. 9, 2013.

[2] C. Kurz, A. Mairani, and K. Parodi, "First experimental-based characterization of oxygen ion beam depth dose distributions at the heidelberg ion-beam therapy center," Physics in Medicine Biology, vol. 57, no. 15, p. 5017, 2012.

[3] D. Schardt, T. Elsässer, and D. Schulz-Ertner, "Heavy-ion tumor therapy: Physical and radiobiological benefits," Rev. Mod. Phys., vol. 82, pp. 383-425, 2010.

[4] "ICRU Report 73-Stoppping of ions heavier than helium," International Commission on Radiation Units and Measurements, 2005.

[5] T. Kanai, N. Matsufuji, T. Miyamoto, J. Mizoe, T. Kamada, H. Tsuji, H. Kato, M. Baba, and H. Tsujii, "Examination of GyE system for HIMAC carbon therapy," International Journal of Radiation Oncology*Biology*Physics, vol. 64, no. 2, pp. $650-656,2006$.

[6] M. Krämer and M. Scholz, "Treatment planning for heavy-ion radiotherapy: calculation and optimization of biologically effective dose," Physics in Medicine and Biology, vol. 45, no. 11, pp. 3319-3330, 2000.

[7] Y. Kase, T. Kannai, Y. Matsumotot, Y. Furusawa, H. Okamato, T. Asaba, M. Sakama, and H. Shinoda, "Mirodosimetric measurements and estimation of human cell survival for heavy-ion beams," Radiation Research Society, vol. 166, pp. 629-638, 2006.

[8] H. Rossi and M. Zaider, Microdosimetry and Its Applications. Springer Berlin Heidelberg, 1996.

[9] http://www.fwt.com/detector/support/LET12_manual.PDF.

[10] L. T. Tran, L. Chartier, D. Bolst, J. Davis, D. A. Prokopovich, A. Pogossov, S. Guatelli, M. I. Reinhard, M. Petasecca, M. L. Lerch, et al., "In-field and out-of-file application in ${ }^{12} \mathrm{C}$ ion therapy using fully 3D silicon microdosimeters," Radiation Measurements, vol. 115, pp. 55-59, 2018.

[11] L. T. Tran, D. Bolst, S. Guatelli, A. Pogossov, M. Petasecca, M. L. Lerch, L. Chartier, D. A. Prokopovich, M. I. Reinhard, M. Povoli, et al., "The relative biological effectiveness for carbon, nitrogen, and oxygen ion beams using passive and scanning techniques evaluated with fully $3 \mathrm{D}$ silicon microdosimeters," Medical physics, vol. 45, no. 5, pp. 2299-2308, 2018.

[12] E. Haettner, H. Iwase, M. Krämer, G. Kraft, and D. Schardt, "Experimental study of nuclear fragmentation of 200 and $400 \mathrm{MeV} / \mathrm{u}^{12} \mathrm{C}$ ions in water for applications in particle therapy," Physics in Medicine and Biology, vol. 58, no. 23, pp. 8265-8279, 2013.

[13] E. Debrot, D. Bolst, B. James, L. Tran, S. Guatelli, M. Petasecca, D. A. Prokopovich, M. Reinhard, N. Matsufuji, M. Jackson, M. Lerch, and A. B. Rosenfeld, "Investigating variable RBE in a ${ }^{12} \mathrm{C}$ minibeam field with microdosimetry and Geant4," 2019.

[14] J. Allison et al., "Geant4 developments and applications," IEEE Transactions on Nuclear Science, vol. 53, no. 1, pp. 270-278, 2006.

[15] S. Agostinelli et al., "Geant4 - a simulation toolkit," Nuclear Instruments and Methods in Physics Research Section A: Accelerators, Spectrometers, Detectors and Associated Equipment, vol. 506, no. 3, pp. $250-$ 303, 2003.

[16] J. Allison et al., "Recent developments in Geant4," Nuclear Instruments and Methods in Physics Research Section A: Accelerators, Spectrometers, Detectors and Associated Equipment, vol. 835, pp. 186 - 225, 2016.

[17] https://www.sintef.no/en/all-laboratories/foundry-laboratory/.

[18] D. Bolst, G. A. Cirrone, G. Cuttone, G. Folger, S. Incerti, V. Ivanchenko, T. Koi, D. Mancusi, L. Pandola, F. Romano, A. B. Rosenfeld, and S. Guatelli, "Validation of Geant4 fragmentation for heavy ion therapy," Nuclear Instruments and Methods in Physics Research Section A: Accelerators, Spectrometers, Detectors and Associated Equipment, vol. 869, pp. 68 - 75, 2017.

[19] D. Bolst, L. T. Tran, S. Guatelli, N. Matsufuji, and A. B. Rosenfeld, "Modelling the biological beamline at HIMAC using Geant4," Journal of Physics: Conference Series, vol. 1154, p. 012003, 2019.

[20] L. T. Tran, L. Chartier, D. A. Prokopovich, D. Bolst, M. Povoli, A. Summanwar, A. Kok, A. Pogossov, M. Petasecca, S. Guatelli, M. I. Reinhard, M. Lerch, M. Nancarrow, N. Matsufuji, M. Jackson, and A. B. Rosenfeld, "Thin silicon microdosimeter utilizing 3-D mems fabrication technology: Charge collection study and its application in mixed radiation fields," IEEE Transactions on Nuclear Science, vol. 65, no. 1, pp. 467-472, 2018.

[21] S. I. Parker, C. J. Kenney, and J. Segal, "3D-a proposed new architecture for solid-state radiation detectors," Nuclear Instruments and Methods in Physics Research Section A: Accelerators, Spectrometers, Detectors and Associated Equipment, vol. 395, no. 3, pp. 328-343, 1997. 
[22] A. Kok, G. Anelli, C. DaVia, J. Hasi, P. Jarron, C. Kenney, J. M. adn S. Parker, J. Segal, S. Watts, and E. Westbrook, "Inactivation of human kidney cells by highenergy monoenergetic heavy-ion beams," Nuclear Instruments and Methods in Physics Research A, vol. 560, pp. 127-130, 2006.

[23] D. Bolst, S. Guatelli, L. Tran, L. Chartier, M. Lerch, N. Matsufuji, and A. Rosenfeld, "Correction factors to convert microdosimetry measurements in silicon to tissue in ${ }^{12} \mathrm{C}$ ion therapy," Physics in Medicine Biology, vol. 62, no. 6, p. 2055, 2017.

[24] R. B. Hawkins, "A statistical theory of cell killing by radiation of varying linear energy transfer," Radiation Research, vol. 140, pp. 366-374, 1994.

[25] K. Chadwick and H. Leenhouts, "A molecular theory of cell survival," Physics in Medicine $E_{3}$ Biology, vol. 18, no. 1, p. 78, 1973.

[26] D. Bolst, Silicon microdosimetry in hadron therapy using Geant4. PhD thesis, University of Wollongong, 2019

[27] T. T. Böhlen, M. Dosanjh, A. Ferrari, and I. Gudowska, "Simulations of microdosimetric quantities with the Monte Carlo code FLUKA for carbon ions at therapeutic energies," International Journal of Radiation Biology, vol. 88, no. 1-2, pp. 176-182, 2012.

[28] G. Battistoni, J. Bauer, T. T. Böhlen, F. Cerutti, M. P. W. Chin, R. Dos Santos Augusto, A. Ferrari, P. G. Ortega, W. Kozłowska, G. Magro, A. Mairani, K. Parodi, P. R. Sala, P. Schoofs, T. Tessonnier, and V. Vlachoudis, "The FLUKA code: An accurate simulation tool for particle therapy," Frontiers in Oncology, vol. 6, p. 116, 2016.

[29] G. Martino, M. Durante, and D. Schardt, "Microdosimetry measurements characterizing the radiation fields of $300 \mathrm{MeV} / \mathrm{u}^{12} \mathrm{C}$ and $185 \mathrm{MeV} / \mathrm{u}^{7} \mathrm{Li}$ pencil beams stopping in water," Physics in Medicine and Biology, vol. 55, no. 12 , pp. 3441-3449, 2010. 